\title{
Global Stability Results in a SVIR Epidemic Model with Immunity Loss Rate Depending on the Vaccine-Age
}

\author{
Raúl Peralta, ${ }^{1,2}$ Cruz Vargas-De-León, ${ }^{3}$ and Pedro Miramontes ${ }^{1,2}$ \\ ${ }^{1}$ Departamento de Matemáticas, Facultad de Ciencias, UNAM, Ciudad Universitaria, Avenida Universidad, 3000 Circuito Exterior, \\ S/N, 04510 Delegación Coyoacán, DF, Mexico \\ ${ }^{2}$ Centro de Ciencias de la Complejidad (C3), Torre de Ingeniería, UNAM, Ciudad Universitaria, Avenida Universidad, \\ 3000 Circuito Exterior, S/N, 04510 Delegación Coyoacán, DF, Mexico \\ ${ }^{3}$ Unidad de Medicina Experimental, Hospital General de México, Dr Balmis No. 148., Colonia Doctores, 06726 México, DF, Mexico
}

Correspondence should be addressed to Cruz Vargas-De-León; leoncruz82@yahoo.com.mx

Received 22 November 2014; Accepted 12 January 2015

Academic Editor: Yanni Xiao

Copyright (C) 2015 Raúl Peralta et al. This is an open access article distributed under the Creative Commons Attribution License, which permits unrestricted use, distribution, and reproduction in any medium, provided the original work is properly cited.

We formulate a susceptible-vaccinated-infected-recovered (SVIR) model by incorporating the vaccination of newborns, vaccineage, and mortality induced by the disease into the SIR epidemic model. It is assumed that the period of immunity induced by vaccines varies depending on the vaccine-age. Using the direct Lyapunov method with Volterra-type Lyapunov function, we show the global asymptotic stability of the infection-free and endemic steady states.

\section{Introduction}

The persistence of protection induced by vaccines plays a central role in the implementation of human vaccination policies. Two types of evidence document the duration of protection induced by a vaccine: on the one hand, immunological parameters such as antibody kinetics over time and on the other hand the disease incidence for which the vaccine was implemented [1]. In this context, measuring the persistence of circulating antibodies is widely used in human vaccines. The specific antibodies induced by the vaccine are the main parameters used for monitoring the duration of the vaccine-induced immune response. This monitor uses population-based studies that compare antibody levels in a protected group with those of a susceptible group, leading to an estimation of disease risk as a function of postvaccination antibody titers. For several vaccines, data resulting from longterm follow-up vaccine studies have been used to create mathematical models to predict the kinetics of antibody persistence over longer periods of time [2]. An example of such modeling results is the recent publication of the duration of protection induced by a human papillomavirus vaccine, while the trials provided data over a 6.4 year follow-up period, the models predicted a long protection [2]. Further, the waning of protection can also be demonstrated by an increased of disease incidence among subjects previously vaccinated. For example, the implementation of a universal varicella vaccination program in the USA in 1995 has resulted in a substantial reduction in morbidity. However, despite this reduction, new cases of varicella continue to occur, mostly in highly vaccinated school communities. Several studies of these cases have suggested that the time since vaccination may be associated with the risk of varicella. Waning of immunity after varicella vaccination in terms of measurable antibodies has been demonstrated to occur in children previously vaccinated. In this regard, the time since vaccination and the age at vaccination were identified as predictors of this loss of immunity [3-5]. The number of vaccine-preventable diseases targeted by routine vaccination schemes is growing, at time, at least 17 vaccine-preventable diseases were targeted by routine vaccination schemes in USA, but potentially all diseases of infectious etiology can be controlled by vaccination schemes and the evaluation of the persistence of vaccine-induced protection with human vaccines is essential for the susceptible population to determine cases of loss protection. The prevention of infection correlates with the induction of specific antibodies. Although loss of antibody after vaccination may render vaccines again 
susceptible to some infections. The time of vaccine-induced immunity may vary according to individual characteristics and the vaccine-age [6]. The vaccination of newborns could have a central role in the evolution of vaccine-induced immunity [7].

In most infectious disease models, it has been assumed that all vaccinated individuals have equal immunity provided by the vaccine during their vaccination period. However, the duration of protection provided by vaccination varies and is influenced by many factors, particularly the individual characteristics and the vaccine-age $[6,7]$. Therefore, there are enough reasons to study the possible effects of loss of vaccineinduced immunity on the dynamics of infectious diseases. Recently, the vaccine-age-dependent epidemic models have been considered [8-10], more precisely, the vaccine-age is used to track the vaccine-protection period and the vaccine wane rate.

Iannelli et al. in [9] developed an SIS epidemic model with super-infection, perfect vaccination and vaccine-age and discussed the asymptotical behavior of the equilibria. $\mathrm{Li}$ et al. in [10] modeled an SIS disease with vaccine-age and treatment rates and exhibited backward bifurcations under some conditions. Duan et al. in [8] formulated an SIR epidemic model with age of vaccination and proved the global stability of the equilibria. In these models the susceptible population is subjected to a vaccination scheme. Here, we will consider that a proportion of individuals get vaccinated immediately after birth or in newborn.

In this paper, by incorporating the vaccination of newborns in vaccine-age and disease related death rate into the SIR model, we formulate a SVIR epidemiological model with a waning rate of vaccine-induced immunity that depends on the vaccine-age. Here, we will analyze the global stability of the steady states in our age-structured model by means of suitable Volterra-type Lyapunov functions of the form:

$$
G(X)=X-1-\ln X \text {. }
$$

Recently, McCluskey and coauthors in $[11,12]$ proved the global stability of the endemic steady state of SIR models with infection-age-dependent by means of Volterra-type Lyapunov functions. Melnik and Korobeinikov [13] used them to establish global stability for infectious diseases models with age-dependent susceptibility. The global-stability problem for basic age-structured models for the transmission of a vectorborne infectious disease is solved using the Volterra-type functions by Vargas-De-León and colleagues [14].

The remainder of this paper is organized as follows. Section 2 is devoted to the model derivation. In Section 3 the existence of steady states is proved and an explicit formula for the reproductive number is derived. In Section 4, we will give a very simple and elegant proof of the global stability of steady states. Section 5 contains the discussion and concluding remarks.

\section{SIR Model with Vaccination of Newborns}

In this section, we introduce an SIR epidemic model and its reformulations. We can assign each vaccinated individual its class age, called vaccine-age and denote it by $\theta$, which is the amount of time that has passed since the moment that initiates the vaccination.

The model divides the total population into the following subgroups that are unvaccinated susceptible individuals $S(t)$, infectious individuals $I(t)$, recovered individuals $R(t)$, and vaccinated individuals $V(t)$. We stratify the vaccinated part of the population $V(t)$ according to vaccine-age

$$
V(t)=\int_{0}^{+\infty} v(\theta, t) d \theta,
$$

where $v(\theta, t)$ denotes the distribution of the vaccinated individuals at time $t$. In this context, then, $\int_{\theta_{1}}^{\theta_{2}} v(\theta, t) d \theta$ is simply the number of vaccinated individuals between vaccine-ages $\theta_{1}$ and $\theta_{2}$ at time $t$.

We assume a simple demographic process in which newly recruited individuals (corresponding to births) enter the population at a rate $\Lambda$ and exit (due to natural death) at a rate $\mu$. A proportion $0<p<1$ of the newly born individuals are vaccinated at birth and appear in the vaccinated class with class age 0 , giving rise to a boundary condition $v(0, t)=$ $p \Lambda$. The parameter $\beta$ is the successful contact rate between infected and noninfected individuals, $\gamma$ is the recovery rate and $\eta$ is the disease related death rate. The function $0<$ $\sigma(\theta) \leq 1$ denotes the vaccine efficacy, which we assume is a decreasing function of vaccine-age, $\theta$. The function $\alpha(\theta)$ denotes the rate at which the vaccine wanes that depends on the vaccine-age (and such vaccinated individuals move to the unvaccinated susceptible class). We assume that $\alpha(\theta)$ and is nonnegative and bounded integrable function.

Combining the above formulations and assumptions, it follows that the model for the transmission dynamics of the disease in the presence of loss of vaccine-induced immunity is given by the following system of nonlinear partial differential equations:

$$
\begin{aligned}
\frac{d S(t)}{d t}= & (1-p) \Lambda-\beta S(t) I(t)-\mu S(t) \\
& +\int_{0}^{+\infty} \alpha(\theta) v(\theta, t) d \theta \\
\frac{d I(t)}{d t}= & \beta S(t) I(t) \\
& +\int_{0}^{+\infty}(1-\sigma(\theta)) \beta v(\theta, t) I(t) d \theta \\
& -(\mu+\gamma+\eta) I(t), \\
& \frac{\partial v(\theta, t)}{\partial t}+\frac{\partial v(\theta, t)}{\partial \theta} \\
& =-(\mu+\alpha(\theta)) v(\theta, t)-(1-\sigma(\theta)) \beta v(\theta, t) I(t),
\end{aligned}
$$

with the following boundary condition $v(0, t)=p \Lambda$ and initial conditions $S(0)=S_{0}>0, I(0)=I_{0}>0$, and $v(\theta, 0)=v_{0}(\theta)>0$, where $S_{0}$ and $I_{0}$ are initial populations of the susceptible individuals and infectious 
individuals, respectively, and an initial distribution of the vaccinated individuals with respect to their vaccine-age $v_{0}(\theta)$ is a Lebesgue integrable function. The equation for $R(t)$ is omitted here.

In the following section, we will discuss existence of steady states, namely, infection-free steady state and endemic steady state. Also, we will derive an explicit formula for the reproductive number.

\section{Steady States and Effective Reproductive Number}

The epidemic systems can have one or two nonnegative steady states. Specifically, the epidemic system always has an infection-free steady state; in this steady state the component of infectious individuals is zero. Also can have an endemic steady state with all components positive.

The system (3) has an infection-free steady state $E_{0}=$ $\left(S^{0}, I^{0}, v^{0}(\theta)\right)$, where

$$
\begin{gathered}
S^{0}=\frac{\Lambda}{\mu}\left(1-p+p \int_{0}^{+\infty} \alpha(\theta) K_{0}(\theta) d \theta\right), \\
I^{0}=0, \quad v^{0}(\theta)=p \Lambda K_{0}(\theta)
\end{gathered}
$$

with

$$
K_{0}(\theta):=\exp \left(-\int_{0}^{\theta}(\mu+\alpha(\tau)) d \tau\right)
$$

$K_{0}(\theta)$ is the survival rate at duration $\theta$ in the vaccinated class.

A possible formula for the reproductive number can be derived by determination of the condition for the instability of infection-free steady state $E_{0}$. Here, we will derive the reproductive number by means of linearization around $E_{0}$, and its condition of instability.

Then we perturb the infection-free steady state by letting

$$
\begin{gathered}
x(t)=S(t)-S^{0}, \quad y(t)=I(t), \\
z(\theta, t)=v(\theta, t)-v^{0}(\theta) .
\end{gathered}
$$

Substituting the variables above in (3) and neglecting terms of order bigger than two, we obtain the following linear system for $x(t), y(t)$, and $z(\theta, t)$ :

$$
\begin{aligned}
& \frac{d x(t)}{d t}=-\beta S^{0} y(t)-\mu x(t)+\int_{0}^{+\infty} \alpha(\theta) z(\theta, t) d \theta, \\
& \frac{d y(t)}{d t}=\beta S^{0} y(t)+\beta y(t) \\
& \quad \cdot \int_{0}^{+\infty}(1-\sigma(\theta)) v^{0}(\theta) d \theta-(\mu+\gamma+\eta) y(t), \\
& \frac{\partial z(\theta, t)}{\partial t}+\frac{\partial z(\theta, t)}{\partial \theta} \\
& =-(\mu+\alpha(\theta)) z(\theta, t)-(1-\sigma(\theta)) \beta v^{0}(\theta) y(t) .
\end{aligned}
$$

We consider exponential solutions of the form:

$$
\begin{gathered}
x(t)=\bar{x} \exp (\lambda t), \quad y(t)=\bar{y} \exp (\lambda t), \\
z(\theta, t)=\bar{z}(\theta) \exp (\lambda t),
\end{gathered}
$$

where $\bar{x}$ and $\bar{y}$ are arbitrary constants, $\bar{z}(\theta)$ is a function of $\theta$, and $\lambda$ is a real or a complex number. Substituting them in system (7), we obtain

$$
\begin{gathered}
\lambda \bar{x}=-\beta S^{0} \bar{y}-\mu \bar{x}+\int_{0}^{+\infty} \alpha(\theta) \bar{z}(\theta) d \theta, \\
\lambda \bar{y}=\beta S^{0} \bar{y}+\beta \bar{y} \int_{0}^{+\infty}(1-\sigma(\theta)) v^{0}(\theta) d \theta-(\mu+\gamma+\eta) \bar{y}, \\
\lambda \bar{z}(\theta)+\frac{\partial \bar{z}(\theta)}{\partial \theta}=-(\mu+\alpha(\theta)) \bar{z}(\theta)-(1-\sigma(\theta)) \beta v^{0}(\theta) \bar{y}, \\
z(t, 0)=0 .
\end{gathered}
$$

If the system (9) has at least one positive eigenvalue then the infection-free steady state $E_{0}$ is unstable. So one eigenvalue of (9) is

$$
\begin{aligned}
\lambda_{1}= & \beta S^{0}+\beta \int_{0}^{+\infty}(1-\sigma(\theta)) v^{0}(\theta) d \theta-(\mu+\gamma+\eta) \\
= & (\mu+\gamma+\eta) \\
& \cdot\left[\frac{\beta}{\mu+\gamma+\eta}\left(S^{0}+\int_{0}^{+\infty}(1-\sigma(\theta)) v^{0}(\theta) d \theta\right)-1\right] .
\end{aligned}
$$

Due to space constraints, we omit the proof that the other two eigenvalues $\left(\lambda_{2}\right.$ and $\left.\lambda_{3}\right)$ of system (9) are negative (or negative real part). Consider

$$
\text { If } \frac{\beta}{\mu+\gamma+\eta}\left(S^{0}+\int_{0}^{+\infty}(1-\sigma(\theta)) v^{0}(\theta) d \theta\right)>1,
$$

then the eigenvalue $\lambda_{1}$ is positive.

Setting

$$
R_{v}:=\frac{\beta S^{0}}{\mu+\gamma+\eta}+\frac{\beta}{\mu+\gamma+\eta} \int_{0}^{+\infty}(1-\sigma(\theta)) v^{0}(\theta) d \theta
$$

yields the expression for the effective reproductive number of the disease. In interpreting this expression, note that $1 /(\mu+\gamma+\eta)$ is the average residence time in the infectious class. The first term $\beta S^{0} /(\mu+\gamma+\eta)$ is the average number of secondary infections produced by a single infectious individual during its infectious period among susceptible population. The second term $\beta \int_{0}^{+\infty}(1-\sigma(\theta)) v^{0}(\theta) d \theta /(\mu+$ $\gamma+\eta)$ is the average number of secondary infections produced by a single infectious individual during its infectious period among vaccinated population. Hence the total expression in (12) represents the average number of secondary infections 
caused by a single infectious individual introduced in an entirely susceptible and vaccinated population, over its entire infectious period, $1 /(\mu+\gamma+\eta)$.

We will now turn to study the conditions of existence of the endemic steady state. System (3) has an endemic steady state $E^{*}=\left(S^{*}, I^{*}, v^{*}(\theta)\right)$, where $S^{*}, I^{*}$, and $v^{*}(\theta)$ satisfy the equalities

$$
\begin{gathered}
0=(1-p) \Lambda-\beta S^{*} I^{*}-\mu S^{*}+\int_{0}^{+\infty} \alpha(\theta) v^{*}(\theta) d \theta, \\
0=\beta S^{*}+\int_{0}^{+\infty}(1-\sigma(\theta)) \beta v^{*}(\theta) d \theta-(\mu+\gamma+\eta), \\
\frac{d v^{*}(\theta)}{d \theta}=-(\mu+\alpha(\theta)) v^{*}(\theta)-(1-\sigma(\theta)) \beta v^{*}(\theta) I^{*}, \\
v^{*}(0)=p \Lambda .
\end{gathered}
$$

From (13), it follows that

$S^{*}$

$$
\begin{aligned}
= & \frac{1}{\mu+\beta I^{*}}\left((1-p) \Lambda+\int_{0}^{+\infty} \alpha(\theta) v^{*}(\theta) d \theta\right) \\
= & \frac{1}{\mu+\beta I^{*}} \\
& \cdot((1-p) \Lambda \\
& \left.\quad+\int_{0}^{+\infty} \alpha(\theta) v^{0}(\theta) \exp \left(-\int_{0}^{\theta}(1-\sigma(\theta)) \beta I^{*} d \tau\right) d \theta\right) .
\end{aligned}
$$

Equations (15) and (16) give

$$
\begin{aligned}
v^{*}(\theta) & =p \Lambda \exp \left(-\int_{0}^{\theta}\left(\mu+\alpha(\tau)+(1-\sigma(\theta)) \beta I^{*}\right) d \tau\right) \\
& =v^{0}(\theta) \exp \left(-\int_{0}^{\theta}(1-\sigma(\theta)) \beta I^{*} d \tau\right) .
\end{aligned}
$$

Combining this with (14), (17), and (18), we obtain the equality

$$
\begin{aligned}
& \frac{\beta}{\mu+\gamma+\eta}\left(S^{*}+\int_{0}^{+\infty}(1-\sigma(\theta)) v^{*}(\theta) d \theta\right) \\
& =\frac{\beta}{(\mu+\gamma+\eta)\left(\mu+\beta I^{*}\right)} \\
& \quad \cdot((1-p) \Lambda \\
& \left.\quad+\int_{0}^{+\infty} \alpha(\theta) v^{0}(\theta) \exp \left(-\int_{0}^{\theta}(1-\sigma(\theta)) \beta I^{*} d \tau\right) d \theta\right) \\
& +\frac{\beta}{\mu+\gamma+\eta}
\end{aligned}
$$

$$
\cdot \int_{0}^{+\infty}(1-\sigma(\theta)) v^{0}(\theta) \exp \left(-\int_{0}^{\theta}(1-\sigma(\theta)) \beta I^{*} d \tau\right) d \theta
$$$$
=1 \text {. }
$$

It is easy to see that function

$$
\begin{aligned}
& H\left(I^{*}\right) \\
& =\frac{\beta}{\mu+\gamma+\eta} \\
& \cdot\left(\frac{1}{\mu+\beta I^{*}}\right. \\
& \quad((1-p) \Lambda \\
& \quad+\int_{0}^{+\infty} \alpha(\theta) v^{0}(\theta) \\
& +\int_{0}^{+\infty}(1-\sigma(\theta)) v^{0}(\theta) \\
& \left.\cdot \exp \left(-\beta I^{*} \int_{0}^{\theta}(1-\sigma(\theta)) d \tau\right) d \theta\right) \\
& \left.\quad\left(-\beta I^{*} \int_{0}^{\theta}(1-\sigma(\theta)) d \tau\right) d \theta\right)
\end{aligned}
$$

is continuous and monotonically decreases with the growth of $I^{*}$ and that equalities

$$
H(0)=R_{v}, \quad \lim _{I^{*} \rightarrow+\infty} H\left(I^{*}\right)=0 \text { hold. }
$$

Hence, for all $R_{v}>1$ there exists $I^{*} \in(0, \infty)$ such that equality $H\left(I^{*}\right)=1$ holds. Corresponding $S^{*}$ and $v^{*}(\theta)$ are defined by (17) and (18), respectively.

Thus, we just proved the following Theorem.

Theorem 1. System (3) always has the infection-free steady state $E_{0}=\left(S^{0}, I^{0}, v^{0}(\theta)\right)$. If $R_{v}>1$, then system also has a unique endemic steady state $E^{*}=\left(S^{*}, I^{*}, v^{*}(\theta)\right)$.

\section{Global Stability of the Steady States}

In this section, our goal is to obtain the global stability results of system (3). We address global asymptotic stability of system (3) by constructing appropriate Volterra-type Lyapunov functions (1). We have the following result.

Theorem 2. (i) When $R_{v} \leq 1$, then the infection-free steady state $E_{0}$ is globally asymptotically stable and is the only steady state. (ii) When $R_{v}>1$, then the endemic steady state $E^{*}$ is globally asymptotically stable. 
Proof. (i) To prove the global asymptotic stability of the infection-free steady state, we consider the following Lyapunov function $L(t)=L_{1}(t)+W(t)$, where

$$
\begin{gathered}
L_{1}(t)=S^{0} G\left(\frac{S(t)}{S^{0}}\right)+I(t), \\
W(t)=\int_{0}^{+\infty}\left[v^{0}(\theta) G\left(\frac{v(\theta, t)}{v^{0}(\theta)}\right)\right] d \theta .
\end{gathered}
$$

Using $\mu S^{0}=(1-p) \Lambda+\int_{0}^{+\infty} \alpha(\theta) v^{0}(\theta) d \theta$, we have

$$
\begin{aligned}
\frac{d L_{1}}{d t} & \\
= & (1-p) \Lambda\left(2-\frac{S(t)}{S^{0}}-\frac{S^{0}}{S(t)}\right) \\
& +\int_{0}^{+\infty} \alpha(\theta) v^{0}(\theta)\left(-\frac{S(t)}{S^{0}}+1+\ln \frac{S(t)}{S^{0}}\right) d \theta \\
& +\int_{0}^{+\infty} \alpha(\theta) v^{0}(\theta)\left(\frac{v(\theta, t)}{v^{0}(\theta)}-\ln \frac{S(t)}{S^{0}}-\frac{S^{0} v(\theta, t)}{S(t) v^{0}(\theta)}\right) d \theta \\
& +\beta I(t) \int_{0}^{+\infty} v^{0}(\theta)(1-\sigma(\theta))\left(\frac{v(\theta, t)}{v^{0}(\theta)}-1\right) d \theta \\
& -(\mu+\gamma+\eta)\left(1-R_{v}\right) I(t) .
\end{aligned}
$$

We have

$$
\begin{aligned}
\frac{d W}{d t}= & \int_{0}^{+\infty}\left(1-\frac{v^{0}(\theta)}{v(\theta, t)}\right) \frac{\partial v(\theta, t)}{\partial t} d \theta \\
=-\int_{0}^{+\infty}\left(1-\frac{v^{0}(\theta)}{v(\theta, t)}\right) & \\
& \left(\frac{\partial v(\theta, t)}{\partial \theta}+(\mu+\alpha(\theta)) v(\theta, t)\right. \\
& +\beta(1-\sigma(\theta)) v(\theta, t) I(t)) d \theta .
\end{aligned}
$$

Note that

$$
\begin{aligned}
& \frac{\partial}{\partial \theta} G\left(\frac{v(\theta, t)}{v^{0}(\theta)}\right) \\
& \quad=\left(1-\frac{v^{0}(\theta)}{v(\theta, t)}\right)\left(\frac{\partial_{\theta} v(\theta, t)}{v^{0}(\theta)}-\frac{v(\theta, t) \cdot D_{\theta}\left[v^{0}(\theta)\right]}{v^{0}(\theta) v^{0}(\theta)}\right),
\end{aligned}
$$

where $\partial_{\theta} v(\theta, t)$ denotes $\partial v(\theta, t) / \partial \theta$, and $D_{\theta}\left[v^{0}(\theta)\right]$ denotes $d v^{0}(\theta) / d \theta$.

We know $D_{\theta}\left[v^{0}(\theta)\right]=-(\mu+\alpha(\theta)) v^{0}(\theta)$.

Hence,

$$
\begin{aligned}
v^{0}(\theta) & \frac{\partial}{\partial \theta} G\left(\frac{v(\theta, t)}{v^{0}(\theta)}\right) \\
& =\left(1-\frac{v^{0}(\theta)}{v(\theta, t)}\right)\left(\partial_{\theta} v(\theta, t)+(\mu+\alpha(\theta)) v(\theta, t)\right) .
\end{aligned}
$$

We have

$$
\begin{aligned}
\frac{d W}{d t}= & -\int_{0}^{+\infty} v^{0}(\theta) \frac{\partial}{\partial \theta} G\left(\frac{v(\theta, t)}{v^{0}(\theta)}\right) d \theta \\
& -\beta I(t) \int_{0}^{+\infty} v^{0}(\theta)(1-\sigma(\theta))\left(\frac{v(\theta, t)}{v^{0}(\theta)}-1\right) d \theta .
\end{aligned}
$$

Hence, using integration by parts,

$$
\begin{aligned}
\int_{0}^{+\infty} & v^{0}(\theta) \frac{\partial}{\partial \theta} G\left(\frac{v(\theta, t)}{v^{0}(\theta)}\right) d \theta \\
= & {\left[v^{0}(\theta) G\left(\frac{v(\theta, t)}{v^{0}(\theta)}\right)\right]_{\theta=0}^{\theta=+\infty} } \\
& -\int_{0}^{+\infty} D_{\theta}\left[v^{0}(\theta)\right] G\left(\frac{v(\theta, t)}{v^{0}(\theta)}\right) d \theta \\
= & {\left.\left[v^{0}(\theta) G\left(\frac{v(\theta, t)}{v^{0}(\theta)}\right)\right]\right]_{\theta=+\infty} } \\
& -v^{0}(0) G\left(\frac{v(0, t)}{v^{0}(0)}\right)-\int_{0}^{+\infty} D_{\theta}\left[v^{0}(\theta)\right] G\left(\frac{v(\theta, t)}{v^{0}(\theta)}\right) d \theta
\end{aligned}
$$

and using the following equalities $v^{0}(0)=p \Lambda, v(0, t)=p \Lambda$, and $D_{\theta}\left[v^{0}(\theta)\right]=-(\mu+\alpha(\theta)) v^{0}(\theta)$, we have

$$
\begin{aligned}
\int_{0}^{+\infty} & v^{0}(\theta) \frac{\partial}{\partial \theta} G\left(\frac{v(\theta, t)}{v^{0}(\theta)}\right) d \theta \\
= & {\left[v^{0}(\theta) G\left(\frac{v(\theta, t)}{v^{0}(\theta)}\right)\right]_{\theta=+\infty} } \\
& +\int_{0}^{+\infty}(\mu+\alpha(\theta)) v^{0}(\theta) G\left(\frac{v(\theta, t)}{v^{0}(\theta)}\right) d \theta .
\end{aligned}
$$

Substituting the expression (29) in (27)

$$
\begin{aligned}
\frac{d W}{d t}= & -\left[v^{0}(\theta) G\left(\frac{v(\theta, t)}{v^{0}(\theta)}\right)\right]_{\theta=+\infty} \\
& -\int_{0}^{+\infty} \mu v^{0}(\theta) G\left(\frac{v(\theta, t)}{v^{0}(\theta)}\right) d \theta \\
& -\int_{0}^{+\infty} \alpha(\theta) v^{0}(\theta)\left[\frac{v(\theta, t)}{v^{0}(\theta)}-1-\ln \frac{v(\theta, t)}{v^{0}(\theta)}\right] d \theta \\
& -\beta I(t) \int_{0}^{+\infty} v^{0}(\theta)(1-\sigma(\theta))\left(\frac{v(\theta, t)}{v^{0}(\theta)}-1\right) d \theta \\
= & -\left[v^{0}(\theta) G\left(\frac{v(\theta, t)}{v^{0}(\theta)}\right)\right]_{\theta=+\infty} \\
& -\int_{0}^{+\infty} \mu v^{0}(\theta) G\left(\frac{v(\theta, t)}{v^{0}(\theta)}\right) d \theta \\
& -\beta I(t) \int_{0}^{+\infty} v^{0}(\theta)(1-\sigma(\theta))\left(\frac{v(\theta, t)}{v^{0}(\theta)}-1\right) d \theta
\end{aligned}
$$




$$
\begin{aligned}
&+\int_{0}^{+\infty} \alpha(\theta) v^{0}(\theta) \\
& \cdot\left[-\frac{v(\theta, t)}{v^{0}(\theta)}+1+\ln \frac{S^{0} v(\theta, t)}{S(t) v^{0}(\theta)}+\ln \frac{S(t)}{S^{0}}\right] d \theta
\end{aligned}
$$

and combining (23) and (30), we have

$$
\begin{aligned}
\frac{d L}{d t}= & \frac{d L_{1}}{d t}+\frac{d W}{d t} \\
= & (1-p) \Lambda\left(2-\frac{S(t)}{S^{0}}-\frac{S^{0}}{S(t)}\right) \\
& -\left[v^{0}(\theta) G\left(\frac{v(\theta, t)}{v^{0}(\theta)}\right)\right]_{\theta=+\infty} \\
& -\int_{0}^{+\infty} \mu v^{0}(\theta) G\left(\frac{v(\theta, t)}{v^{0}(\theta)}\right) d \theta \\
& -\int_{0}^{+\infty} \alpha(\theta) v^{0}(\theta)\left[G\left(\frac{S(t)}{S^{0}}\right)+G\left(\frac{S^{0} v(\theta, t)}{S(t) v^{0}(\theta)}\right)\right] d \theta \\
& -(\mu+\gamma+\eta)\left(1-R_{v}\right) I(t) .
\end{aligned}
$$

Therefore, $R_{v} \leq 1$ ensures that $d L / d t \leq 0$ holds in the nonnegative region of the phase space. The strict equality holds only if $S(t)=S^{0}$ and $v(\theta, t)=v^{0}(\theta)$ holds simultaneously with either $R_{v}=1$ or $I(t)=0$. It is easy to verify that infection-free steady state $E_{0}$ is the only invariant set of the system in this set, and hence by LaSalle's invariance principle for semiflows (Corollary 2.3 in [15]), $R_{v} \leq 1$ is sufficient to ensure that this steady state is globally asymptotically stable.

(ii) To prove the global asymptotic stability of the endemic steady state, we consider the following Volterra-type Lyapunov function $U(t)=U_{1}(t)+J(t)$, where

$$
\begin{aligned}
& U_{1}(t)=S^{*} G\left(\frac{S(t)}{S^{*}}\right)+I^{*} G\left(\frac{I(t)}{I^{*}}\right), \\
& J(t)=\int_{0}^{+\infty}\left[v^{*}(\theta) G\left(\frac{v(\theta, t)}{v^{*}(\theta)}\right)\right] d \theta .
\end{aligned}
$$

Using $\beta S^{*}+\beta \int_{0}^{+\infty}(1-\sigma(\theta)) v^{*}(\theta) d \theta=(\mu+\gamma+\eta)$ and $\mu S^{*}=$ $(1-p) \Lambda-\beta S^{*} I^{*}+\int_{0}^{+\infty} \alpha(\theta) v^{*}(\theta) d \theta$, we have

$$
\begin{aligned}
\frac{d U_{1}}{d t}= & (1-p) \Lambda\left(2-\frac{S(t)}{S^{*}}-\frac{S^{*}}{S(t)}\right) \\
& +\int_{0}^{+\infty} \alpha(\theta) v^{*}(\theta)\left(-\frac{S(t)}{S^{*}}+1+\ln \frac{S(t)}{S^{*}}\right) d \theta \\
& +\int_{0}^{+\infty} \alpha(\theta) v^{*}(\theta) \\
& \cdot\left(\frac{v(\theta, t)}{v^{*}(\theta)}-\ln \frac{S(t)}{S^{*}}-\frac{S^{*} v(\theta, t)}{S(t) v^{*}(\theta)}\right) d \theta
\end{aligned}
$$

$$
\begin{aligned}
+\beta I^{*} \int_{0}^{+\infty} & v^{*}(\theta)(1-\sigma(\theta)) \\
& \cdot\left(\frac{v(\theta, t) I(t)}{v^{*}(\theta) I^{*}}-\frac{v(\theta, t)}{v^{*}(\theta)}-\frac{I(t)}{I^{*}}+1\right) d \theta .
\end{aligned}
$$

Similarly, we have

$$
\begin{gathered}
\frac{d J}{d t}=\int_{0}^{+\infty}\left(1-\frac{v^{*}(\theta)}{v(\theta, t)}\right) \frac{\partial v(\theta, t)}{\partial t} d \theta \\
=-\int_{0}^{+\infty} v^{*}(\theta)\left(\frac{v(\theta, t)}{v^{*}(\theta)}-1\right) \\
\cdot\left(\frac{\partial_{\theta} v(\theta, t)}{v(\theta, t)}+\mu+\alpha(\theta)\right. \\
+\beta(1-\sigma(\theta)) I(t)) d \theta \\
=\int_{0}^{+\infty} v^{*}(\theta)\left(\frac{v(\theta, t)}{v^{*}(\theta)}-1\right) \\
\cdot\left(\frac{\partial_{\theta} v(\theta, t)}{v(\theta, t)}+\mu+\alpha(\theta)+\beta(1-\sigma(\theta)) I^{*}\right) d \theta \\
+\beta I^{*} \int_{0}^{+\infty} v^{*}(\theta)(1-\sigma(\theta)) \\
\cdot\left(\frac{v(\theta, t)}{v^{*}(\theta)}-1-\frac{v(\theta, t) I(t)}{v^{*}(\theta) I^{*}}+\frac{I(t)}{I^{*}}\right) d \theta .
\end{gathered}
$$

Note that

$$
\begin{aligned}
& \frac{\partial}{\partial \theta} G\left(\frac{v(\theta, t)}{v^{*}(\theta)}\right) \\
& \quad=\left(1-\frac{v^{*}(\theta)}{v(\theta, t)}\right)\left(\frac{\partial_{\theta} v(\theta, t)}{v^{*}(\theta)}-\frac{v(\theta, t) \cdot D_{\theta}\left[v^{*}(\theta)\right]}{v^{*}(\theta) v^{*}(\theta)}\right) .
\end{aligned}
$$

We know $D_{\theta}\left[v^{*}(\theta)\right]=-(\mu+\alpha(\theta)) v^{*}(\theta)-(1-\sigma(\theta)) \beta v^{*}(\theta) I^{*}$. Hence,

$$
\begin{aligned}
v^{*}(\theta) & \frac{\partial}{\partial \theta} G\left(\frac{v(\theta, t)}{v^{*}(\theta)}\right) \\
= & \left(1-\frac{v^{*}(\theta)}{v(\theta, t)}\right) \\
& \cdot\left(\partial_{\theta} v(\theta, t)+\left(\mu+\alpha(\theta)+(1-\sigma(\theta)) \beta I^{*}\right) v(\theta, t)\right) .
\end{aligned}
$$


We have

$$
\begin{aligned}
\frac{d J}{d t}= & -\int_{0}^{+\infty} v^{*}(\theta) \frac{\partial}{\partial \theta} G\left(\frac{v(\theta, t)}{v^{*}(\theta)}\right) d \theta \\
+\beta I^{*} \int_{0}^{+\infty} v^{*}(\theta)(1-\sigma(\theta)) & \\
& \cdot\left(\frac{v(\theta, t)}{v^{*}(\theta)}-1-\frac{v(\theta, t) I(t)}{v^{*}(\theta) I^{*}}+\frac{I(t)}{I^{*}}\right) d \theta .
\end{aligned}
$$

Hence, using integration by parts,

$$
\begin{aligned}
\int_{0}^{+\infty} & v^{*}(\theta) \frac{\partial}{\partial \theta} G\left(\frac{v(\theta, t)}{v^{*}(\theta)}\right) d \theta \\
= & {\left[v^{*}(\theta) G\left(\frac{v(\theta, t)}{v^{*}(\theta)}\right)\right]_{\theta=0}^{\theta=+\infty} } \\
& -\int_{0}^{+\infty} D_{\theta}\left[v^{*}(\theta)\right] G\left(\frac{v(\theta, t)}{v^{*}(\theta)}\right) d \theta \\
= & {\left[v^{*}(\theta) G\left(\frac{v(\theta, t)}{v^{*}(\theta)}\right)\right]_{\theta=+\infty} } \\
& -v^{*}(0) G\left(\frac{v(0, t)}{v^{*}(0)}\right)-\int_{0}^{+\infty} D_{\theta}\left[v^{*}(\theta)\right] G\left(\frac{v(\theta, t)}{v^{*}(\theta)}\right) d \theta
\end{aligned}
$$

and using the following equalities $v^{*}(0)=p \Lambda, v(0, t)=p \Lambda$, and $D_{\theta}\left[v^{*}(\theta)\right]=-\mu v^{*}(\theta)-\alpha(\theta) v^{*}(\theta)-(1-\sigma(\theta)) \beta v^{*}(\theta) I^{*}$. we have

$$
\begin{gathered}
\int_{0}^{+\infty} v^{*}(\theta) \frac{\partial}{\partial \theta} G\left(\frac{v(\theta, t)}{v^{*}(\theta)}\right) d \theta \\
=\left[v^{*}(\theta) G\left(\frac{v(\theta, t)}{v^{*}(\theta)}\right)\right]_{\theta=+\infty} \\
+\int_{0}^{+\infty}\left(\mu+\alpha(\theta)+(1-\sigma(\theta)) \beta I^{*}\right) \\
\cdot v^{*}(\theta) G\left(\frac{v(\theta, t)}{v^{*}(\theta)}\right) d \theta .
\end{gathered}
$$

Substituting the expression (39) in (37)

$$
\begin{aligned}
\frac{d J}{d t}= & -\left[v^{*}(\theta) G\left(\frac{v(\theta, t)}{v^{*}(\theta)}\right)\right]_{\theta=+\infty} \\
& -\int_{0}^{+\infty}(\mu+\alpha(\theta)) v^{*}(\theta) G\left(\frac{v(\theta, t)}{v^{*}(\theta)}\right) d \theta \\
& +\beta I^{*} \int_{0}^{+\infty} v^{*}(\theta)(1-\sigma(\theta)) \\
& \cdot\left(\ln \frac{v(\theta, t)}{v^{*}(\theta)}-\frac{v(\theta, t) I(t)}{v^{*}(\theta) I^{*}}+\frac{I(t)}{I^{*}}\right) d \theta
\end{aligned}
$$

and combining (33) and (40), we have

$$
\begin{aligned}
\frac{d U}{d t}= & \frac{d U_{1}}{d t}+\frac{d J}{d t} \\
= & (1-p) \Lambda\left(2-\frac{S(t)}{S^{*}}-\frac{S^{*}}{S(t)}\right) \\
& -\left[v^{*}(\theta) G\left(\frac{v(\theta, t)}{v^{*}(\theta)}\right)\right]_{\theta=+\infty} \\
& -\int_{0}^{+\infty}\left(\mu+(1-\sigma(\theta)) \beta I^{*}\right) v^{*}(\theta) G\left(\frac{v(\theta, t)}{v^{*}(\theta)}\right) d \theta \\
& -\int_{0}^{+\infty} \alpha(\theta) v^{*}(\theta) \\
& \cdot\left[G\left(\frac{S(t)}{S^{*}}\right)+G\left(\frac{S^{*} v(\theta, t)}{S(t) v^{*}(\theta)}\right)\right] d \theta .
\end{aligned}
$$

That is positive-definite function $U(t)$ has nonpositive derivative $d U / d t$. Furthermore, the equality $d U / d t=0$ holds only if $S(t)=S^{*}$ and $v(\theta, t)=v^{*}(\theta)$, simultaneously. Thus the endemic steady state $E^{*}$ is the only positively invariant subset of the set with $d U / d t=0$, and hence by LaSalle's invariance principle for semiflows (Corollary 2.3 in [15]), the steady state $E^{*}$ is globally asymptotically stable when it exists.

The following Corollary immediately follows from the proof.

\section{Corollary 3. System (3) is uniformly persistent.}

\section{Concluding Remarks}

The vaccination of newborn or neonatal immunization may be an effective preventive strategy against some pathogens. The ideal vaccine would be a single dose given at birth and providing immediate and long protection against multiple diseases. In this paper, we introduced and analytically studied the global stability properties of an SIR epidemiological model with vaccination, where it is assumed that the waning rate of vaccine-induced immunity is depending on the vaccine-age. In this model, we introduce the vaccination of newborns or neonatal immunization in the vaccine-age.

One of the fundamental questions of mathematical epidemiology is to find the reproductive number, we derived an explicit formula for the effective reproductive number of epidemic system (3) using the condition that the infectionfree steady state $E_{0}$ is unstable if and only if $R_{v}>1$.

We perform a nonlinear stability analysis, by means of the Lyapunov function techniques and LaSalle's Invariance Principle for semiflows. We show that the classical threshold condition for the effective reproductive number, $R_{v}$, holds: $R_{v}>1$; then the endemic steady $E^{*}$ is globally asymptotically stable, whereas if $R_{v} \leq 1$, then the infection-free steady state $E_{0}$ is globally asymptotically stable.

It is noteworthy, that we only impose integrability condition on the wane rate function of vaccine $\alpha(\theta)$ in the model, and hence, global stability results derived are robust and do 
not depend on specific forms of this function. Some forms of the function $\alpha(\theta)$ have been proposed in $[8,9]$. The following function $\alpha(\theta)$ is reported in [9]:

$$
\alpha(\theta)= \begin{cases}0, & \text { if } 0<\theta \leq \theta_{\min }, \\ \alpha_{0}, & \text { if } \theta_{\min }<\theta<\theta_{\max },\end{cases}
$$

where $\theta_{\min }$ and $\theta_{\max }$ are minimum and maximum vaccineages, respectively, and $\alpha_{0}$ is positive constant. We generalized the specific form given in [8]

$$
\alpha(\theta)=\left\{\begin{array}{l}
0, \\
\quad \text { if } 0<\theta \leq \theta_{\min }, \\
\kappa_{0}\left(\theta-\theta_{\min }\right)^{2} \exp \left[-\kappa_{1}\left(\theta-\theta_{\min }\right)\right], \\
\quad \text { if } \theta_{\text {min }}<\theta \leq \theta_{\text {med }}, \\
\alpha_{0}, \\
\quad \text { if } \theta_{\text {med }}<\theta<\theta_{\text {max }},
\end{array}\right.
$$

where $\kappa_{0}$ and $\kappa_{1}$ are positive constants and $\theta_{\text {med }}$ is intermedium vaccine-age.

We notice that the effective reproductive number can be written as

$$
\begin{aligned}
R_{v}=R_{0}(1-p & \\
& \left.\cdot\left(1-\int_{0}^{+\infty}(\alpha(\theta)+\mu(1-\sigma(\theta))) K_{0}(\theta) d \theta\right)\right),
\end{aligned}
$$

where $R_{0}=\beta \Lambda /(\mu(\mu+\gamma+\eta))$ is the basic reproductive number of the disease. From this, a measure of vaccine impact (see [16]), $\phi$, can be derived as

$$
\begin{aligned}
\phi & =\frac{R_{0}-R_{v}}{R_{0}} \\
& =p\left(1-\int_{0}^{+\infty}(\alpha(\theta)+\mu(1-\sigma(\theta))) K_{0}(\theta) d \theta\right) .
\end{aligned}
$$

It is easy to check that

$$
\frac{\partial \phi}{\partial p}=1-\int_{0}^{+\infty}(\alpha(\theta)+\mu(1-\sigma(\theta))) K_{0}(\theta) d \theta>0 .
$$

Thus, the vaccine impact $\phi$ is an increasing function with respect to the argument $p$.

Finally, we note that the approach used in this paper can be applied to a wider variety of the age-dependent epidemic models with waning vaccine-induced immunity.

\section{Conflict of Interests}

The authors declare that they have no conflict of interests.

\section{Acknowledgments}

Raúl Peralta is thankful to the Postdoctoral fellowship from DGAPA, UNAM. The authors would like to thank anonymous reviewers for improving this paper with their comments and suggestions.

\section{References}

[1] E. Vidor, "Evaluation of the persistence of vaccine-induced protection with human vaccines," Journal of Comparative Pathology, vol. 142, supplement 1, pp. S96-S101, 2010.

[2] M. Aregay, Z. Shkedy, G. Molenberghs, M.-P. David, and F. Tibaldi, "Model-based estimates of long-term persistence of induced HPV antibodies: a flexible subject-specific approach," Journal of Biopharmaceutical Statistics, vol. 23, no. 6, pp. 12281248, 2013.

[3] S. A. Plotkin, "Correlates of protection induced by vaccination," Clinical and Vaccine Immunology, vol. 17, no. 7, pp. 1055-1065, 2010.

[4] S. A. Plotkin, "Complex correlates of protection after vaccination," Clinical Infectious Diseases, vol. 56, no. 10, pp. 1458-1465, 2013.

[5] S. S. Chaves, P. Gargiullo, J. X. Zhang et al., "Loss of vaccineinduced immunity to varicella over time," The New England Journal of Medicine, vol. 356, no. 11, pp. 1121-1129, 2007.

[6] M. Prelog, "Differential approaches for vaccination from childhood to old age," Gerontology, vol. 59, no. 3, pp. 230-239, 2013.

[7] N. Wood and C.-A. Siegrist, "Neonatal immunization: where do we stand?" Current Opinion in Infectious Diseases, vol. 24, no. 3, pp. 190-195, 2011.

[8] X. Duan, S. Yuan, and X. Li, "Global stability of an SVIR model with age of vaccination," Applied Mathematics and Computation, vol. 226, pp. 528-540, 2014.

[9] M. Iannelli, M. Martcheva, and X.-Z. Li, "Strain replacement in an epidemic model with super-infection and perfect vaccination," Mathematical Biosciences, vol. 195, no. 1, pp. 23-46, 2005.

[10] X.-Z. Li, J. Wang, and M. Ghosh, "Stability and bifurcation of an SIVS epidemic model with treatment and age of vaccination," Applied Mathematical Modelling, vol. 34, no. 2, pp. 437-450, 2010.

[11] P. Magal, C. C. McCluskey, and G. F. Webb, "Lyapunov functional and global asymptotic stability for an infection-age model," Applicable Analysis, vol. 89, no. 7, pp. 1109-1140, 2010.

[12] C. C. McCluskey, "Delay versus age-of-infection-global stability," Applied Mathematics and Computation, vol. 217, no. 7, pp. 3046-3049, 2010.

[13] A. V. Melnik and A. Korobeinikov, "Lyapunov functions and global stability for SIR and SEIR models with age-dependent susceptibility," Mathematical Biosciences and Engineering, vol. 10, no. 2, pp. 369-378, 2013.

[14] C. Vargas-De-León, L. Esteva, and A. Korobeinikov, "Agedependency in host-vector models: the global analysis," Applied Mathematics and Computation, vol. 243, pp. 969-981, 2014.

[15] K. Mischaikow, H. Smith, and H. R. Thieme, "Asymptotically autonomous semiflows: chain recurrence and Lyapunov functions," Transactions of the American Mathematical Society, vol. 347, no. 5, pp. 1669-1685, 1995.

[16] E. H. Elbasha, "Global stability of equilibria in a two-sex HPV vaccination model," Bulletin of Mathematical Biology, vol. 70, no. 3, pp. 894-909, 2008. 


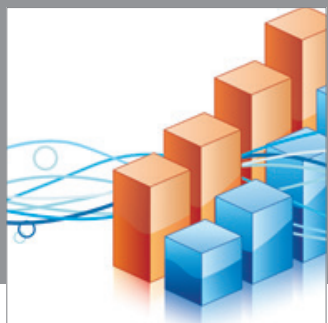

Advances in

Operations Research

mansans

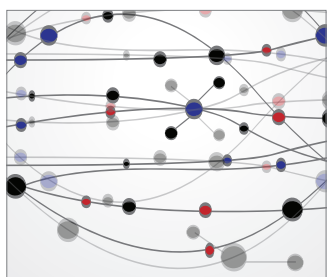

The Scientific World Journal
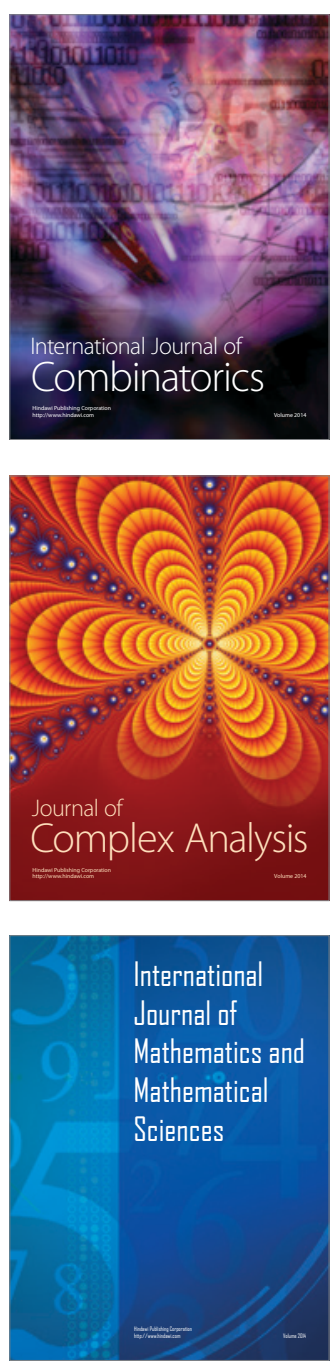
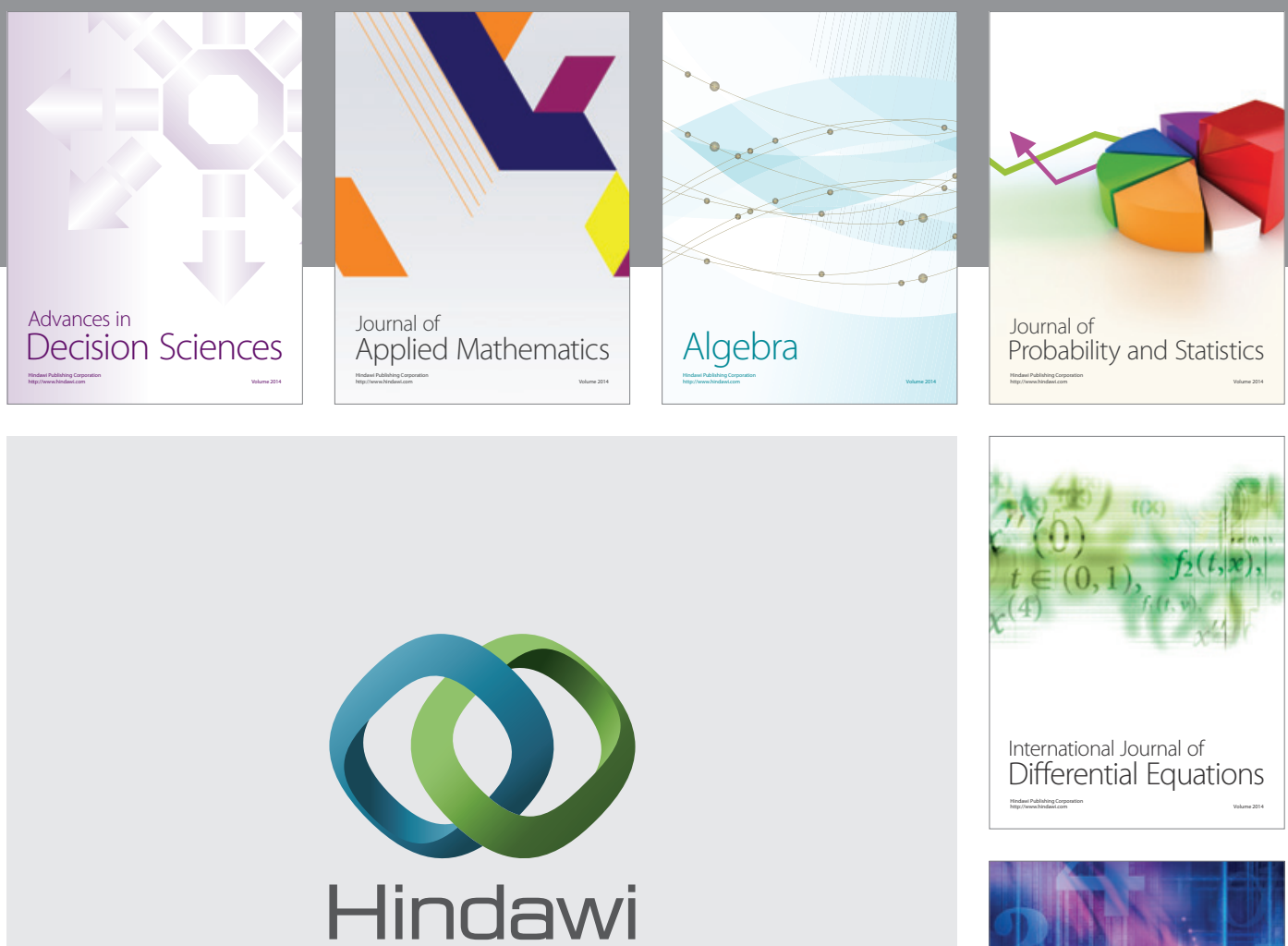

Submit your manuscripts at http://www.hindawi.com
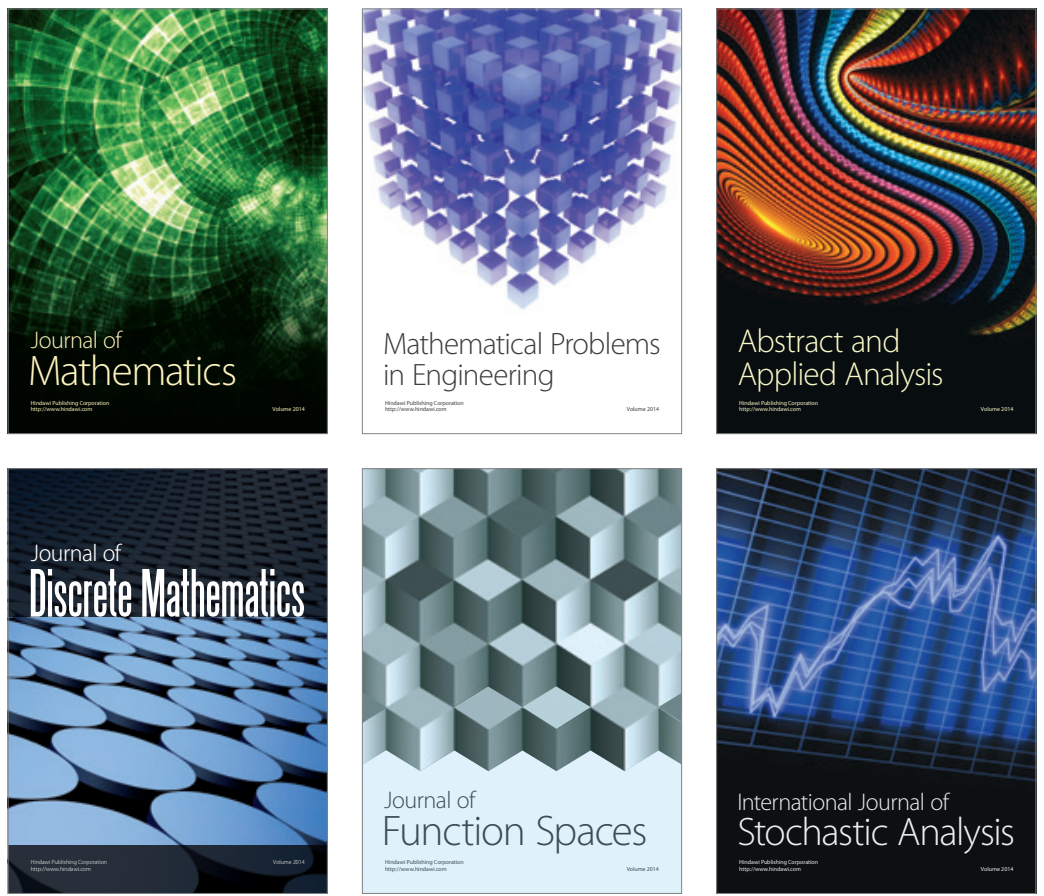

Journal of

Function Spaces

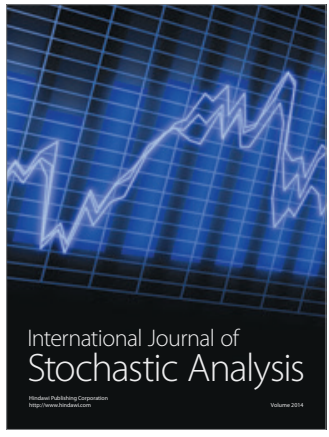

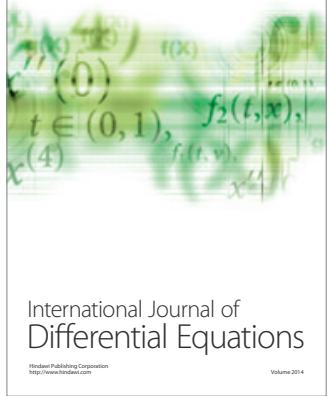
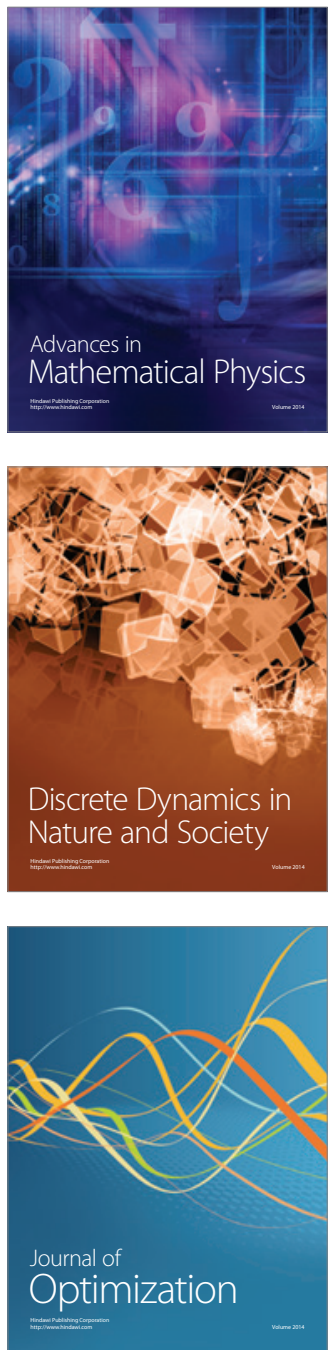